\title{
Microscopy of Semiconducting Materials 1985
}

\author{
Anthony G. Cullis
}

RSRE Corporation

The fourth in a biennial series on Microscopy of Semiconducting Materials was held at St. Catherine's College, Oxford, England, on March 27-29, 1985. The chairmen of the conference were Dr. A. G. Cullis (RSRE, Malvern) and Dr. D. B. Holt (Imperial College, London). The event was organized under the auspices of the Royal Microscopical Society and cosponsored by the Institute of Physics (UK) and the Materials Research Society. More than 200 delegates attended from the UK and 14 other countries. The applications of various types of microscopy and related characterization techniques to semiconducting materials studies spanned a wide range which encompassed both fundamental solid-state research and also advanced electronic device work. While much of the work reported in the 85 conference papers involved the study of elemental silicon, extensive coverage was also given to compound semiconductors.

The conference was opened by Prof. Sir Peter Hirsch. The different scientific sessions focused attention on individual topics of particluar current importance and were introduced by invited speakers. A paper by Dr. F. A. Ponce (Xerox Labs) reviewed the application of high resolution transmission electron microscopy to the study of microdefects in silicon, gallium arsenide, and II-VI semiconducting compounds. The use of lattice imaging techniques to identify nanometer-size crystal defects was described. Following papers extended the application of these techniques to problems involving the diffusion and precipitation of impurities in silicon, the structure of grain boundaries in germanium, and the characterization of compound semiconductor epitaxial layers and superlattices. A special session was devoted to dislocation phenomena with an initial presentation by Prof. P. Haasen (Gottingen University) outlining the effects of stage IV compression in elemental semiconductors. Contributed work covered additional experimental and theoretical progress in this area.

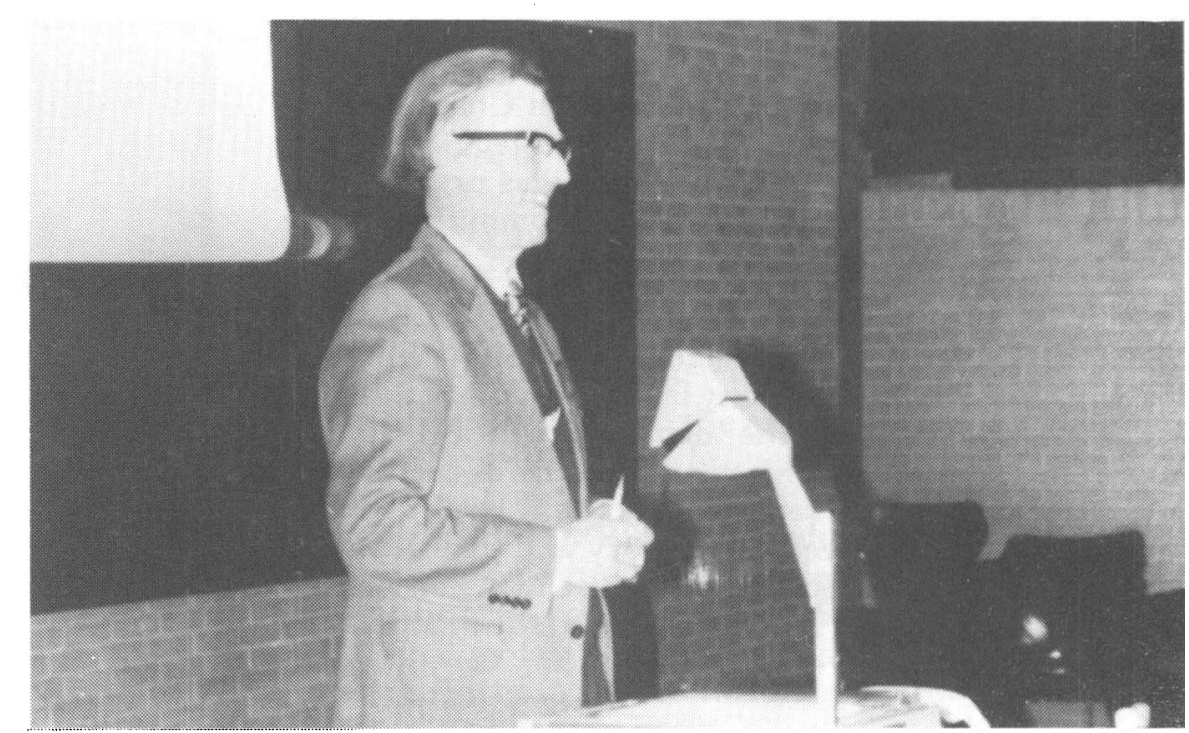

G. R. Booker answers questions on developments in TEM studies.

An overview of developments in transmission electron microscope studies of compound semiconductors was given by Dr. G. R. Booker (Oxford University). The session following this wide-ranging talk covered important EL2 defect phenomena, grain boundaries, and the effects of heavy ion damage in gallium arsenide. The characterization of defects in indium phosphide and cadmium telluride was also treated. A large amount of work focused on the investigation of epitaxial layer structures. Several papers discussed alloy clustering and spinodal decomposition effects in ternary and quaternary III-V semiconductor layers. The importance of lattice relaxation in thin specimens was emphasized. A number of transmission microscope studies of the structure of superlattices based on gallium arsenide and lead telluride demonstrated the layer growth characteristics found in these systems.

The growth of epitaxial silicon was also described with detailed consideration of both homoepitaxy and silicon deposited on sapphire and gallium arsenide. Work showing the nature of silicide layers on silicon was reported and Dr. J. M. Gibson (A T\&T Bell Labs) discussed the influence of interfacial atomic structure on Schottky barrier height. Contributed papers described the variety of defect structures found in deposited nickel, titanium, and tungsten silicide films.

The application of special techniques in the scanning electron microscope was covered in a session opened by Prof. J. Heydenreich (East German Academy of Sciences) who treated advances in the characterization of semiconductor defects by electron beam induced conductivity and scanning deep level transient spect roscopy techniques. Other speakers described a range of applications and theoretical work on the induced conductivity and also scanning electron acoustic microscopy techniques. Further presentations placed some emphasis on cathodoluminescence studies of bulk and ion-implanted gallium arsenide and zinc selenide.

Applications of microanalysis with special reference to the scanning transmission electron microscope were presented by Dr. L. M. Brown (Cambridge University), and other workers covered analysis ranging from the use of Auger electrons to $\mathrm{Z}$-contrast imaging of dopant distributions in silicon. Prof. J. W. Mayer (Cornell University) described a detailed study of silicides and lateral diffusion couples by combined microscopy and ion backscattering techniques: indeed, his inimitable, enter- 
taining style of presentation made a profound impression upon the audience. Recent developments in atom probe microanalysis of semiconductors were covered in another paper which demonstrated the atomic layer depth resolution of the technique.

$X$-ray topography and diffraction studies of semiconductors were treated in a session introduced by Dr. B. K. Tanner (Durham University). The main emphasis of the work described focused on III-V compounds, and the various authors in the session covered such topics as the measurement of dislocation mobilities and electronic device studies by techniques including synchrotron $x$-ray topography and double crystal diffractometry.

Transient processing of semiconductors was a further important conference topic, and Dr. B. Bensahel (CNET, Grenoble) reviewed the fabrication of single crystal silicon-oninsulator by transient melting techniques. This subject $w$ as also considered by other authors who, in addition, gave a detailed model for defect formation in the layers. The effects of rapid thermal annealing upon ionimplanted silicon and gallium arsenide was another topic described in a number of microscopy-based studies. Conventional device processing steps in troduce a range of defects and microscopic structures into semiconductors and the application of transmission electron microscopy, particularly of crosssectional samples, for the support of VLSI silicon tech nology was addressed by Dr. C. Claeys (IMEC, Heverlee) and Dr. H. Oppolzer (Siemens Research Labs). This theme was continued in contributed papers which covered studies ranging from work on oxidationinduced stacking faults and ion implantation damage to measurements of dopant distributions and investigations of buried nitride layers formed by nitrogen ion implantation.

The conference closed with a session on device testing by use of scanning electron beam techniques and recent advances in this area were surveyed by Dr. S. M. Davidson (Lintech Instruments). Several other papers continued the discussion and included consideration of electron beam damage in finished transistors and the measurement of VLSI pattern linewidths.

The formal schedule of the three-day conference was fairly intensive, although there were no parallel sessions so that delegates could attend all presentations of interest. In addition to the oral paper sessions, a poster session was held on the evening of March 25 and generated a lot of useful scientific interaction.

The proceedings of the conference are being published by the UK Institute of Physics as Number 76 in their Conference Series. The volume can be obtained from the Publications Office at Techno House, Redcliffe Way, Bristol BS1 6NX, UK.

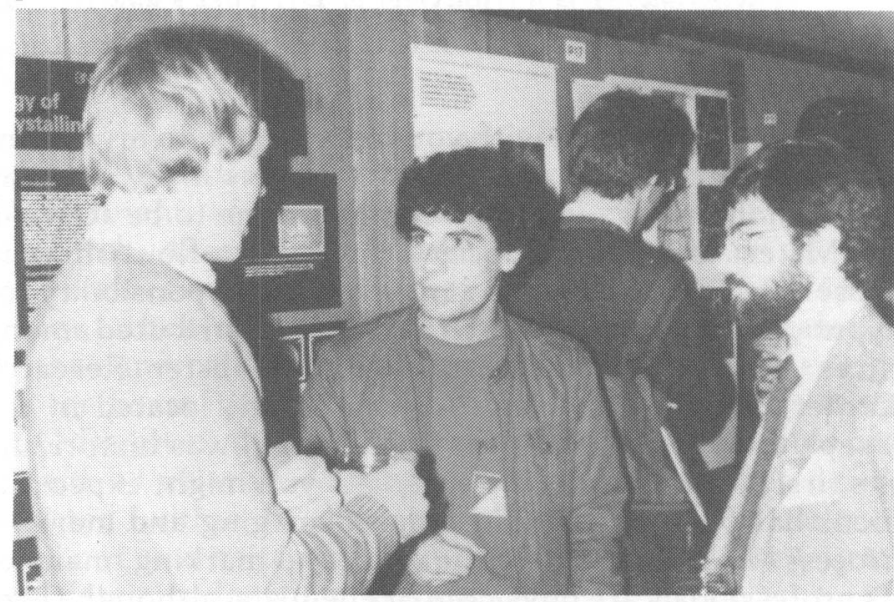

Delegates discuss poster displays during March 25 poster session.

\section{Fractals to be Topic of Gordon Research Conference}

A Gordon Research Conference on Fractals will be held July 15-19, 1985 at Hawthorne College, Antrim, NH. The conference is chaired by Benoit $B$. Mandelbrot, IBM Research Center, whoco-chaired with Dann Passoja the highly successful symposium, Fractal Aspects of Materials: Metal and Catalyst Surfaces, Powders and Aggregates, at the 1984 MRS Fall Meeting. Vice chairm an of the Gordon Conference is Ray Orbach, UCLA.

The program consists of the following lectures:

Diffraction from Fractal Dust (Michael V. Berry)

Self-Avoiding Walks on Fractal Lattices (Jean Vannimenus)

Universality in the Mandelbrot Set of Mode Blockings (Itamar Procaccia)

Fractal Structure of Some Old and New Materials (James E. Martin)

Anomalous Diffusion in Random Fractals (Amnon Aharony)
Fractal Approach to Random Superconductor Networks (Antonio Coniglio)

Diffusion and Force: Force Correlations on Random Fractals (Yuval Gefen)

Hierarchial Interactions and Effective Dimensional Lowering (Nihat Berker)

Properties of Fractal Surfaces (Shlomo Alexander)

Reaction Kinetics on Fractals: Experiments and Simulations (Raoul Kopelman)

Fractals from Energy Transfer Studies (Mostafa A. El Sayed)

Direct Energy Transfer on Fractal Structures (Uri Even)

Stochastic Flows on Lattices of Fractal Valences (John J. Kozak)

Chemical Reactions on Fractal Structures (Joseph Klafter)

Earthquakes: Their Fractal Aspects in Time and Space (Geoffrey King)
Multiplicative Chaos and the Mandelbrot Measures (Jacques Peyriere)

Fractals Generated by the Dynamics of Iteration (Michael Barnsley)

Lattice Green's Function Approach to Diffusion Limited Aggregation (Harvey Sher)

Phase Transitions and Mandelbrot Sets (Hans-Otto Peitgen)

Fractal Dimension and Asymptotic Degrees of Freedom in Turbulence (Cyprian Foias)

Fractal Aspects of Turbulence (Erik Siggia)

Fractal Time and Molecular Motion in Polymers, Glasses and Proteins (John Bendler)

Attendance is limited to 100 persons. Interested scientists may obtain the required application form from the Director of the Gordon Research Conferences, University of Rhode Island, Kingston, RI 02281-0801. The conference fee is $\$ 275$. 